\title{
ON ELLIPTIC CURVES WHOSE CONDUCTOR IS A PRODUCT OF TWO PRIME POWERS
}

\author{
MOHAMMAD SADEK
}

Abstract. We find all elliptic curves defined over $\mathbb{Q}$ that have a rational point of order $N, N \geq 4$, and whose conductor is of the form $p^{a} q^{b}$, where $p, q$ are two distinct primes and $a, b$ are two positive integers. In particular, we prove that Szpiro's conjecture holds for these elliptic curves.

\section{INTRODUCTION}

Let $E$ be an elliptic curve defined over $\mathbb{Q}$ with minimal discriminant $\Delta_{E}$. We define the conductor $N_{E}$ of $E$ to be

$$
N_{E}=\prod_{p \mid \Delta_{E}} p^{f_{p}}, f_{p}=\operatorname{ord}_{p}\left(\Delta_{E}\right)-m_{E}+1,
$$

where $m_{E}$ is the number of components on the special fiber of the Néron model of $E$ defined over $\mathbb{F}_{p}, f_{p} \geq 1$; see ([13], Chapter IV, $\left.\S 10,11\right)$. Furthermore, $f_{p}=1$ if and only if $E$ has multiplicative reduction at $p$. We recall that $N_{E}$ and $\Delta_{E}$ have the same prime divisors.

The problem of finding all elliptic curves $E$ defined over $\mathbb{Q}$ of a given conductor has been investigated in many articles. Ogg produced the complete list of elliptic curves whose conductor is a 2 -power or $2^{a} 3^{b}$; see [9] and [10. A series of papers has dealt with the problem under the condition that $E$ has a rational torsion point. For example, in [7] the elliptic curves with conductor $p^{m}, p$ is prime, and 2-torsion points were listed.

It was shown in 6 that all elliptic curves with conductor $2^{m} p^{n}$ where $p \equiv 3$ or $5 \bmod 8, p \neq 3$, that have a rational point of order 2 , are effectively determined under the truth of the conjecture of Ankeny-Artin-Chowla.

It is worth mentioning that the complete list of elliptic curves with a prime conductor has already been produced. The following theorem gives this list explicitly.

Theorem 1.1 (Theorem 5.3.2, 11]). Let $E$ be an elliptic curve over $\mathbb{Q}$ with prime conductor $p$. Then either $\left|\Delta_{E}\right|=p$ or $p^{2}$, or else $p=11$ and $\Delta_{E}=11^{5}$, or $p=17$ and $\Delta_{E}=17^{4}$, or $p=19$ and $\Delta_{E}=19^{3}$, or $p=37$ and $\Delta_{E}=37^{3}$. In particular, $\Delta_{E} \mid p^{5}$.

The elliptic curves in Theorem 1.1 turn out to satisfy Szpiro's conjecture, which is stated below for the convenience of the reader.

Conjecture 1.2. If $E$ is an elliptic curve over $\mathbb{Q}$, then

$$
\left|\Delta_{E}\right| \ll_{\epsilon} N_{E}^{6+\epsilon} \text {. }
$$

Received by the editor February 27, 2012.

2010 Mathematics Subject Classification. Primary 14H52.

(C)2013 American Mathematical Society Reverts to public domain 28 years from publication 
One of the popular strategies to find elliptic curves $E / \mathbb{Q}$ with a given conductor is to solve certain Diophantine equations obtained by equating the discriminant of $E$ to the product of powers of the prime divisors of the conductor.

Mazur gave a complete classification of the torsion subgroup $E_{\text {tors }}(\mathbb{Q})$ of $E(\mathbb{Q})$; see (Theorem $7.5, \S 8$, Chapter VIII, $[12]$ ). More precisely, $E_{\text {tors }}(\mathbb{Q})$ is isomorphic to one of the following fifteen groups:

$$
\mathbb{Z} / n \mathbb{Z}, 1 \leq n \leq 12, n \neq 11 ; \text { or } \mathbb{Z} / 2 \mathbb{Z} \times \mathbb{Z} / 2 n \mathbb{Z}, 1 \leq n \leq 4
$$

Given that $P \in E(\mathbb{Q})[m], m \neq 2,3$, it is known that there exist $b, c \in \mathbb{Q}$ such that the following Weierstrass equation defines an elliptic curve $E_{b, c}$ isomorphic to $E$ :

$$
E_{b, c}: y^{2}+(1-c) x y-b y=x^{3}-b x^{2}
$$

with the image of $P$ being $(0,0)$. The discriminant $\Delta(b, c)$ of $E_{b, c}$ is given as follows:

$$
\Delta(b, c)=b^{3}\left(16 b^{2}-b\left(8 c^{2}+20 c-1\right)-c(1-c)^{3}\right) .
$$

By taking $m$ to be an integer in $\{4,5,6,7,8,9,10,12\}$, one finds an explicit relation between $b, c$; see for example $\S 2$ of 8 .

In this article, we generalize Theorem 1.1 to elliptic curves whose conductors have two distinct prime divisors only. More precisely, we generate the list of all elliptic curves with $\mathbb{Q}$-rational torsion points of order $N, N \geq 4$, whose conductor is a product of two prime powers.

Now we give a brief outline for our approach to solve the problem. As we have seen, the family of all elliptic curves with a rational point of order $N$ can be classified using a universal Weierstrass equation. Moreover, we can use certain transformations to write integral Weierstrass equations for the elliptic curves $E_{b, c}$. Since the prime divisors of the minimal discriminant are exactly those of the conductor, we equate the produced discriminant to a product of two prime powers. Consequently, the problem is reduced to solving one or several Diophantine equations.

In fact, the Diophantine equations we produce are more subtle when $N \in\{4,5\}$, whereas the corresponding Diophantine equations are elementary when $N \geq 6$. We collect the harder Diophantine equations in $\$ 2$ for more convenience. Several techniques are followed to attack these equations, including elementary methods, factorization over number fields, properties of Lucas sequences, and well-known results from the literature.

Each family of elliptic curves with rational points of order $N$ is treated separately. Given an $N, 4 \leq N \leq 12, N \neq 11$, we list all elliptic curves with a $\mathbb{Q}$-rational $N$-torsion point such that the conductor has only two distinct prime divisors. Moreover, we find a constant $K>0$ such that given such an elliptic curve $E$, the absolute value of the minimal discriminant $\Delta_{E}$ of $E$ is bounded above by the $K$-th power of the conductor $N_{E}$. In particular, we prove Szpiro's conjecture for these families of elliptic curves. When $N=10,12$, we show that there are no elliptic curves with an $N$-torsion point whose conductor is a product of two prime powers.

\section{Diophantine equations}

Catalan's Conjecture (now referred to as Mihăilescu's Theorem) will appear several times in this article, so we prefer to state it. 
Proposition 2.1 (Mihăilescu's Theorem). The only integer solution to the Diophantine equation $x^{m}-y^{n}=1$, where $m, n>1$, is $(x, m, y, n)=( \pm 3,2,2,3)$.

Now we start solving some Diophantine equations that we will use to prove our main results.

Lemma 2.2. There are no integer solutions $(p, m, y, n)$ to the equation

$$
16 p^{m}+1=y^{n}
$$

where $|p|, n$ are prime integers, $m>1$, and $y=l^{t}$, where $|l|$ is prime and $t>0$.

Proof. Let $(p, m, y, n)$ be such a solution to $16 p^{m}+1=y^{n}$. We observe that $y$ is odd. Furthermore, $|p| \neq 2$, otherwise we will have a Catalan solution $\left|y^{n}-2^{m+4}\right|=1$. So $|p|$ is odd.

We assume $n$ is an odd prime. So $p^{m} y>0$. Since

$$
16 p^{m}=y^{n}-1=(y-1)\left(y^{n-1}+y^{n-2}+\ldots+y+1\right),
$$

where the first factor is even, the second factor is the sum of $n$ odd terms, and hence is odd. Therefore $16 \mid y-1$.

i. If $\operatorname{gcd}\left(y-1, \frac{y^{n}-1}{y-1}\right)=1$, then either $y-1=16 p^{m}$ and $\frac{y^{n}-1}{y-1}=1$, which yield no solutions, or $y=17$ and $\frac{y^{n}-1}{y-1}=17^{n-1}+\ldots+17+1=p^{m}$. The latter equation is not solvable for $n \geq 3, m \geq 2$; see Corollary 1 in [2]. The last possible value $y=-15$ is rejected because it is not a prime power.

ii. If $p \mid \operatorname{gcd}\left(y-1, \frac{y^{n}-1}{y-1}\right)$, then $y \equiv 1 \bmod p$. Moreover, $y-1= \pm 16 p^{h}$, $h>0$, and $\frac{y^{n}-1}{y-1}= \pm p^{m-h}$. This implies that $n=|p|$ ( $n$ is prime). We observe that

$$
\begin{aligned}
\pm p^{m-h} & =\sum_{i=0}^{n-1}\left(1 \pm 16 p^{h}\right)^{i}=\sum_{i=0}^{n-1} \sum_{j=0}^{i}\left(\begin{array}{l}
i \\
j
\end{array}\right)\left( \pm 16 p^{h}\right)^{j} \\
& =p+\sum_{i=1}^{n-1} \sum_{j=1}^{i}\left(\begin{array}{l}
i \\
j
\end{array}\right)\left( \pm 16 p^{h}\right)^{j} \\
& =p \pm 16 p^{h} n(n-1) / 2+\sum_{i=1}^{n-1} \sum_{j=2}^{i}\left(\begin{array}{l}
i \\
j
\end{array}\right)\left( \pm 16 p^{h}\right)^{j}
\end{aligned}
$$

Since $n=|p|$, one has $m-h=1$. If we consider the positive sign, the above equality is $p=p+L$, and $L>0$, a contradiction. Otherwise, the above equality is

$$
-2 p=-16 p^{h} p(p-1) / 2+\sum_{i=1}^{n-1} \sum_{j=2}^{i}\left(\begin{array}{l}
i \\
j
\end{array}\right)\left(-16 p^{h}\right)^{j},
$$

a contradiction.

Assume $n=2$. Then $16 p^{m}=y^{2}-1=(y-1)(y+1)$. If $p \mid \operatorname{gcd}(y-1, y+$ 1 ), then $p=2$ (a contradiction, as then we will have a Catalan solution, $y^{2}-$ $\left.2^{m+4}=1\right)$. Otherwise, we either have $y+1= \pm 2^{\alpha} p^{m}$ and $y-1= \pm 2^{4-\alpha}$, so $y \in\{17,9,5,3,2,0,-1,-3,-7,-15\}$ with $p^{m}=5,3,14$ corresponding to $y=$ 
$9,-7,-15$, or $y-1= \pm 2^{\alpha} p^{m}$ and $y+1= \pm 2^{4-\alpha}$, so $y=7,-17,-9$ with $p^{m}=$ $3,18,5$. These solutions are rejected.

Lemma 2.3 (Lemma 5.5, 3]). The only positive integer solutions $(x, y, h, n)$ to the equation

$$
x^{2}+2^{h}=y^{n}, n>1, y \text { odd, } h>2,
$$

are $(x, y, h, n)=(7,3,5,4)$ and $(x, y, n)=\left(2^{h-2}-1,2^{h-2}+1,2\right)$.

Now we use some techniques in elementary number theory to find the integer solutions of some Diophantine equations.

Lemma 2.4. The integer solutions $(x, y, l), l>1, y>0$, to the Diophantine equation

$$
x^{2}-125= \pm 4 y^{l}
$$

are

$$
\{( \pm 15,5,2),( \pm 63,31,2),( \pm 11,1, l),( \pm 5,5,2),( \pm 25,5,3)\},
$$

or $l$ is odd, $5 \nmid x$, and $x^{2}-125=4 y^{l}$.

Proof. We consider many possibilities:

i. $x^{2}-125=-4 y^{l}$ : By investigating perfect squares of the form $125-4 \lambda$, one finds the following possible solutions:

$$
\{( \pm 11,1, l),( \pm 9,11,1),( \pm 7,19,1),( \pm 5,5,2),( \pm 3,29,1),( \pm 1,31,1)\} .
$$

ii. $l=2 k$ and $x^{2}-125=4 y^{l}$ : Then we can write $\left(x-2 y^{k}\right)\left(x+2 y^{k}\right)=$ 125. Therefore, we can assume that $\left(x-2 y^{k}\right) \in\{ \pm 1, \pm 5, \pm 25, \pm 125\}$. Consequently, $l=2$ and we have

\begin{tabular}{|c|c|c|c|c|}
\hline$x-2 y^{k}$ & \pm 1 & \pm 5 & \pm 25 & \pm 125 \\
\hline$(x, y)$ & $( \pm 63, \pm 31)$ & $( \pm 15, \pm 5)$ & $( \pm 15, \mp 5)$ & $( \pm 63, \mp 31)$ \\
\hline
\end{tabular}

iii. $l$ is odd, $x^{2}-125=4 y^{l}$, and $5 \mid x$ : Then $5 \mid y$. Since $l \geq 3$, one has $25 \mid x$. In fact, $l=3$ and 5||$y$. Dividing by 125 , one has $5(x / 25)^{2}-$ $1=4(y / 5)^{3}$. If $(x, y)$ is an integer solution to the latter equation, then $(X, Y)=(100(x / 25), 20(y / 5))$ is a solution to $X^{2}-k=Y^{3}, k=2000$. In [5], all of Mordell's equations with $|k| \leq 10000$ were solved in $\mathbb{Z}$. In fact, the only solutions of $X^{2}-2000=Y^{3}$ are $( \pm 100,20)$ and $( \pm 44,-4)$. Therefore, the only integer solution of $x^{2}-125=4 y^{l}, l$ odd, and $5 \mid x$ is $( \pm 25,5,3)$.

iv. $l$ is odd, $x^{2}-125=4 y^{l}$, and $5 \nmid x$ : We notice that in (1), both $x$ and $y$ are odd. In fact, this equation has a finite number of integer solutions.

Corollary 2.5. The only integer solutions $(s, y, l)$ of the Diophantine equation

$$
s^{2}-11 s-1= \pm y^{l},
$$

where $|s|, y>0$ are prime powers, $l>1$, are

$$
\{(13,5,2),(-2,5,2),(37,31,2),(11,1, l),(8,5,2),(3,5,2),(-7,5,3)\},
$$

or $l$ is odd and $s^{2}-11 s-1=y^{l}$. 
Proof. After completing the square, one has

$$
x^{2}-125= \pm 4 y^{l}, \text { where } x=2 s-11 .
$$

According to Lemma 2.4, we obtain the above triples. We observe that the triples $(-26,31,2)$ and $(18,5,3)$, corresponding to $(x, y, l)=(-63,31,2)$ and $(25,5,3)$, are rejected, because $|s|$ is not a prime power.

\section{Elliptic CURVES With RATIONAL $n$-TORSION POINTS}

Let $E / \mathbb{Q}$ be an elliptic curve with minimal discriminant $\Delta_{E}$ and conductor $N_{E}$. Given that $P \in E(\mathbb{Q})[m], m \geq 4$, there exist $b, c \in \mathbb{Q}$ such that the following Weierstrass equation defines an elliptic curve $E_{b, c}$ isomorphic to $E$ :

$$
E_{b, c}: y^{2}+(1-c) x y-b y=x^{3}-b x^{2},
$$

with the image of $P$ being $(0,0)$. The invariants $c_{4}(b, c)$ and $\Delta(b, c)$ of $E_{b, c}$ are as follows:

$$
\begin{aligned}
& c_{4}(b, c)=16 b^{2}+8 b(1-c)(c+2)+(1-c)^{4}, \\
& \Delta(b, c)=b^{3}\left(16 b^{2}-b\left(8 c^{2}+20 c-1\right)-c(1-c)^{3}\right) .
\end{aligned}
$$

By taking $m$ to be an integer in $\{4,5,6,7,8,9,10,12\}$, one obtains an explicit relation between $b, c$; see $\S 2$ of [8].

3.1. Case $n=4$. Assuming that $P \in E(\mathbb{Q})[4]$, one has that $c=0$ in (2). We set $\lambda:=b$. The following Weierstrass equation describes $E$ :

$$
E: y^{2}+x y-\lambda y=x^{3}-\lambda x^{2} .
$$

Assume $\lambda=\frac{s}{t}, s, t \in \mathbb{Z}, \operatorname{gcd}(s, t)=1$. We obtain an integral Weierstrass equation describing $E$ using the change of variables $x \mapsto x / t^{2}, y \mapsto y / t^{3}$. This integral equation is

$$
E \quad: \quad y^{2}+t x y-s t^{2} y=x^{3}-s t x^{2}
$$

with the following invariants:

$$
\begin{aligned}
\Delta_{E} & =s^{4} t^{7}(16 s+t), \\
c_{4} & =t^{2}\left(16 s^{2}+16 s t+t^{2}\right), \\
c_{6} & =-t^{3}\left(-64 s^{3}+120 s^{2} t+24 s t^{2}+t^{3}\right) .
\end{aligned}
$$

Theorem 3.1. Let $E / \mathbb{Q}$ be an elliptic curve such that $E(\mathbb{Q})[4] \neq\{0\}$. Assume moreover that $N_{E}=p q$, where $p \neq q$ are primes. Then $\left|\Delta_{E}\right|=p^{\alpha} q^{\beta}$ is given as follows:

$$
2^{4} \times 3,2^{4} \times 5,2^{4} \times 3^{7}, 2^{8} \times 7,2^{8} \times 3^{2}, 2^{8} \times 7^{7}, 3^{2} \times 7,3^{2} \times 5^{2}, \text { and },
$$

\begin{tabular}{|c||l|c|c|c|c|c|c|c|c|}
\hline$\left|\Delta_{E}\right|$ & $2^{2 k+4} p$ & $2^{2 k+4} p^{4}$ & $2^{4 k} p$ & $2^{4 k} p^{7}$ & $p^{4} q^{b}$ & $p^{4} q^{7 b}$ & $p^{4 k} q$ & $p^{4 k} q^{7}$ & $p^{2 k} q$ \\
\hline$p, q$ & $p=2^{k-4} \pm 1, k \geq 4$ & $p=2^{k+4} \pm 1, k>0$ & $16 p \pm 1=q^{b}, b>1$ & $q=16 p^{k} \pm 1, k>1$ & $q=p^{2 k}+16, k>0$ \\
\hline
\end{tabular}

Proof. Let $s, t \in \mathbb{Z}$ be such that $E$ is given by the following Weierstrass equation:

$$
E: y^{2}+t x y-s t^{2} y=x^{3}-s t x^{2}, \text { where } \Delta_{E}=s^{4} t^{7}(16 s+t) .
$$

One has $\operatorname{gcd}(s, t)=\operatorname{gcd}(s, 16 s+t)=1$, and $\operatorname{gcd}(t, 16 s+t)=2^{k}$, where $0 \leq k \leq 4$; otherwise $2^{k-4} \mid s$, which is a contradiction. In fact, if $k>1$, then the Weierstrass equation is not minimal at 2. Moreover, if $\operatorname{ord}_{p}(t)$ is odd, then $E$ has additive reduction at $p$. 
We first treat the case that $\left|\Delta_{E}\right|=2^{a} p^{b}$, where $p$ is an odd prime, and $a, b>0$. Given $s$ and $t$, the following table gives the possible values for $\Delta_{E}=s^{4} t^{7}(16 s+t)=$ $2^{\alpha} p^{\beta}$. Observe that the table includes all possible values for $\Delta_{E}$, even when $E$ has additive reduction at some prime divisor of $t$.

\begin{tabular}{|c|c|c|c|c|}
\hline$|t||s|$ & $2^{m}$ & $2^{m} p^{n}$ & $p^{n}$ & 1 \\
\hline $2^{k}, k>4$ & - & - & $\begin{array}{c}-2^{5} \times 3^{8}, 2^{3} \times 3^{4} \\
\pm p^{4} 2^{7 k+4}, p=2^{k-4} \pm 1\end{array}$ & $\begin{array}{c}2^{5} \times 3^{2}, 2^{3} \times 3 \\
\pm 2^{7 k+4} p, p=2^{k-4} \pm 1\end{array}$ \\
\hline $2^{k}, 1 \leq k \leq 4$ & - & - & - & $\begin{array}{c}2^{8} \times 3^{2},-2^{8} \times 7 \\
-2^{4} \times 3,2^{4} \times 5 \\
\end{array}$ \\
\hline $2^{k} p^{l}, k>4$ & - & - & - & - \\
\hline $2^{k} p^{l}, 1 \leq k \leq 4$ & - & - & - & $\begin{array}{l}2^{8} \times 3^{2}, \pm 2^{8} \times 7^{7} \\
\pm 2^{4} \times 3^{7}, 2^{4} \times 5^{7}\end{array}$ \\
\hline$p^{l}$ & $\pm 2^{4 m} p^{7}, p=2^{4+m} \pm 1$ & - & - & - \\
\hline 1 & $\pm 2^{4 m} p, p=2^{4+m} \pm 1$ & - & - & - \\
\hline
\end{tabular}

When $(|s|,|t|)=\left(2^{m}, p^{l}\right)$, we need to find solutions to $|16 s+t|=\left|2^{4+m} \pm p^{l}\right|=1$. Therefore, we either have the unique Catalan solution or $l=1$; see Proposition 2.1. The same reasoning and coprimality give the remaining possible values of $\Delta_{E}$ in the above table. Recall that if $\operatorname{ord}_{p}(t)>1$, then $E$ is not minimal at $p$, and we should consider $\operatorname{ord}_{p}\left(\Delta_{E}\right) \bmod 12$.

Now we assume $2 \nmid N_{E}$. So without loss of generality we can assume that $\operatorname{gcd}(t, 16 s+t)=1$. Therefore, at least one of $|s|,|t|,|16 s+t|$ is 1 .

Case $|s|=1$. Then $\left|\Delta_{E}\right|=\left|t^{7}(t \pm 16)\right|$. Assuming $N_{E}=p q$, one observes that if $|t|=p^{a}, a>1$, then $E$ is not minimal at $p$. In fact, if $a \equiv 1 \bmod 2$, then after minimizing $E$ we obtain that $\operatorname{ord}_{p}\left(c_{4}\right)=2 \neq 0$, and hence $E$ has additive reduction at $p$ contradicting the fact that $p \| N_{E}$. Therefore, we assume $|t|=p^{2 a}$ and $\left|\Delta_{E}\right|=p^{2 a}\left|p^{2 a} \pm 16\right|=p^{2 a} q^{b}$. Thus we need to solve $\left|p^{2 a} \pm 16\right|=q^{b}$. Lemma 2.3 gives the solution $(p, a, q, b>1)=( \pm 3,1, \pm 5,2)$ to the equation $p^{2 a}+16=q^{b}$, and $\Delta_{E}=3^{2} \times 5^{2}$. A simple factorization argument shows that the only solution to $p^{2 a}-16=q^{b}$ is $( \pm 5,1, \pm 3,2)$, and $\Delta_{E}=3^{2} \times 5^{2}$. The solution to $16-p^{2 a}=q^{b}$ is $( \pm 3,1,7,1)$, with $\Delta_{E}=3^{2} \times 7$.

Case $|t|=1$. Then $\left|\Delta_{E}\right|=\left|s^{4}(16 s \pm 1)\right|=p^{4 a} q^{b}$; in other words, $|16 s \pm 1|=$ $\left|16 p^{a} \pm 1\right|=q^{b}$. According to Lemma 2.2 $a=1$ or $b=1$, and so $\left|\Delta_{E}\right|=p^{4} q^{b}$ or $p^{4 a} q$ respectively.

Case $|16 s+t|=1$. Then we want to solve $16 p^{m}=q^{n} \pm 1$, and $\Delta_{E}=s^{4} t^{7}$. Again, according to Lemma 2.2. $\left|\Delta_{E}\right|=p^{4 m} q^{7}$ or $p^{4} q^{7 n}$.

Theorem 3.2. Let $E / \mathbb{Q}$ be an elliptic curve such that $E(\mathbb{Q})[4] \neq\{0\}$. Assume moreover that $N_{E}=p q$, where $p \neq q$ are primes. Then $\left|\Delta_{E}\right|<N_{E}^{32}$. In particular, $E / \mathbb{Q}$ satisfies Szpiro's conjecture.

Proof. We only need to check that $\left|\Delta_{E}\right|<N_{E}^{32}$ for the values of $\left|\Delta_{E}\right|$ given in Theorem 3.1. This is straightforward for the first row of possible values of $\left|\Delta_{E}\right|$ appearing in Theorem 3.1. In fact, $\left|\Delta_{E}\right| \leq N_{E}^{8}$. 
Now we are going to verify that $\left|\Delta_{E}\right|<N_{E}^{32}$ for the values of $\left|\Delta_{E}\right|$ in the table of Theorem 3.1 .

$$
\begin{aligned}
\left|\Delta_{E}\right| & =2^{2 k+4} p^{m}=2^{12} 2^{2(k-4)}\left(2^{k-4} \pm 1\right)^{m}<2^{12}\left[2 \times\left(2^{k-4} \pm 1\right)\right]^{2}\left(2^{k-4} \pm 1\right)^{m} \\
& <2^{14}\left(2^{k-4} \pm 1\right)^{2+m}<2^{10}\left(2^{k-4} \pm 1\right)^{6+m} \leq N_{E}^{10}, \text { where } m=1,4 \\
\left|\Delta_{E}\right| & =2^{4 k} p^{m}=2^{4 k}\left(2^{k+4} \pm 1\right)^{m}<\left(2^{k+4} \pm 1\right)^{4}\left(2^{k+4} \pm 1\right)^{m}=p^{m+4}<N_{E}^{11} \\
& \text { where } m=1,7 \\
\left|\Delta_{E}\right| & =p^{4} q^{m b}=p^{4}(16 p \pm 1)^{m}<p^{4} \times p^{4 m}=p^{4 m+4}<N_{E}^{4 m+4}, \text { where } m=1,7, \\
\left|\Delta_{E}\right| & =p^{4 k} q^{m}=p^{4 k}\left(16 p^{k} \pm 1\right)^{m}<\left(16 p^{k} \pm 1\right)^{4}\left(16 p^{k} \pm 1\right)^{m}=q^{m+4}<N_{E}^{m+4} \\
& \text { where } m=1,7 \\
\left|\Delta_{E}\right| & =p^{2 k} q=p^{2 k}\left(p^{2 k}+16\right)<\left(p^{2 k}+16\right)^{2}=q^{2}<N_{E}^{2} .
\end{aligned}
$$

3.2. Case $n=5$. Assuming that $P \in E(\mathbb{Q})[5]$, one has that $b=c$ in (2). Set $\lambda=b$. Then the following Weierstrass equation describes $E$ :

$$
E: y^{2}+(1-\lambda) x y-\lambda y=x^{3}-\lambda x^{2} .
$$

Assume $\lambda=\frac{s}{t}, s, t \in \mathbb{Z}$. We can obtain an integral Weierstrass equation describing $E$ using the following change of variables: $x \mapsto x / t^{2}, y \mapsto y / t^{3}$. This integral equation is

$$
E \quad: \quad y^{2}+(t-s) x y-s t^{2} y=x^{3}-s t x^{2},
$$

where the invariants of $E$ are given by

$$
\begin{aligned}
\Delta_{E} & =s^{5} t^{5}\left(s^{2}-11 s t-t^{2}\right), \\
c_{4} & =24 s t^{2}(-s+t)+\left(s^{2}-6 s t+t^{2}\right)^{2} .
\end{aligned}
$$

Theorem 3.3. Let $E / \mathbb{Q}$ be an elliptic curve such that $E(\mathbb{Q})[5] \neq\{0\}$. Assume moreover that $N_{E}=p^{\alpha} q^{\beta}$, where $p \neq q$ are primes, and $\alpha, \beta>0$. Then $\left|\Delta_{E}\right|=p^{a} q^{b}$ is given as follows:

$$
2^{5} \times 5^{2}, 2^{15} \times 5^{2}, 3^{5} \times 5^{2}, 13^{5} \times 5^{2}, 37^{5} \times 31^{2}, 7^{5} \times 5^{3}, p^{5 k} q^{2 l+1} .
$$

Proof. As we saw above there exist $s, t \in \mathbb{Z}$ such that $E$ is given by the equation

$$
E: y^{2}+(t-s) x y-s t^{2} y=x^{3}-s t x^{2} .
$$

The assumption that $N_{E}$ is a product of two distinct prime powers together with the fact that $\operatorname{gcd}(t, s)=\operatorname{gcd}\left(s, s^{2}-11 s t-t^{2}\right)=\operatorname{gcd}\left(t, s^{2}-11 s t-t^{2}\right)=1$ imply that at least one of $|s|,|t|,\left|s^{2}-11 s t-t^{2}\right|$ is 1 .

Case $|t|=1$. Then one has $\Delta_{E}=s^{5}\left(s^{2} \mp 11 s-1\right)$. Consequently $s$ and $s^{2} \mp 11 s-$ 1 are both prime powers. Now we are going to solve the Diophantine equation $s^{2} \mp 11 s-1= \pm y^{l}$ and spot out the integer solutions $(s, y, l)$ where $s, y$ are prime powers. Completing the square, we need to find the integer solutions of

$$
x^{2}-125= \pm 4 y^{l}, \text { where } x=2 s \mp 11 .
$$


The solutions of the latter Diophantine equation is given in Lemma 2.4. In fact, we obtain the following table:

$$
\begin{array}{|c|c||c|c|}
(x, s, y, l) & \Delta_{E} & (x, s, y, l) & \Delta_{E} \\
( \pm 15, \pm 13,5,2) & \pm 13^{5} \times 5^{2} & ( \pm 25, \pm 7,5,3) & \pm 7^{5} \times 5^{3} \\
( \pm 15, \pm 2,5,2) & \pm 2^{5} \times 5^{2} & \left( \pm 5, \pm 2^{3}, 5,2\right) & \pm 2^{15} \times 5^{2} \\
( \pm 63, \pm 37,31,2) & \pm 37^{5} \times 31^{2} & ( \pm 5, \mp 3,5,2) & \mp 3^{5} \times 5^{2} \\
( \pm 63, \pm 26,31,2) & \pm 26^{5} \times 31^{2} & (x, s, q, 1),|s|=p^{k} & \pm p^{5 k} q
\end{array}
$$

or $\left|\Delta_{E}\right|=p^{5 k} q^{2 l+1}$ for finitely many possible primes $q$.

Case $|s|=1$. Then $\Delta_{E}=t^{5}\left(1 \mp 11 t-t^{2}\right)$. Similarly, we need to solve the Diophantine equation $125-x^{2}= \pm 4 y^{l}$, where $x=2 t \pm 11$. In fact, we obtain the same values given in the above table.

Case $\left|s^{2}-11 s t-t^{2}\right|=1$. Thus $|s|=p^{m},|t|=q^{n}$ and $\left|\Delta_{E}\right|=p^{5 m} q^{5 n}$. Now we complete the square and have that $\left|\left(2 p^{m} \mp 11 q^{n}\right)^{2}-125 q^{2 n}\right|=4$. Any solution to the latter equation will yield a solution to the Diophantine equation $x^{2}-125 y^{2}= \pm 4$, where $x=2 p^{m} \mp 11 q^{n}$ and $y=q^{n}$. We will start solving $x^{2}-125 y^{2}=-4$, which is a Pell's equation for which we have the solution $(11,1)$. Thus any other solution $(x, y)$ is given by

$$
\frac{x+y \sqrt{125}}{2}= \pm\left(\frac{11+\sqrt{125}}{2}\right)^{k}
$$

see for example Proposition 6.3.16 in 4]. One has

$$
\begin{gathered}
\frac{y}{2}=\frac{ \pm 1}{2^{k}}\left(\left(\begin{array}{l}
k \\
1
\end{array}\right) 11^{k-1}+\left(\begin{array}{l}
k \\
3
\end{array}\right) 11^{k-3} \times 125+\ldots+\left(\begin{array}{c}
k \\
1
\end{array}\right) 11 \times 125^{(k-2) / 2}\right), \text { if } k \text { is even, } \\
\frac{x}{2}=\frac{ \pm 1}{2^{k}}\left(11^{k}+\left(\begin{array}{l}
k \\
2
\end{array}\right) 11^{k-2} \times 125+\left(\begin{array}{l}
k \\
4
\end{array}\right) 11^{k-4} \times 125^{2}\right. \\
\left.+\ldots+\left(\begin{array}{c}
k \\
1
\end{array}\right) 11 \times 125^{(k-1) / 2}\right), \text { if } k \text { is odd. }
\end{gathered}
$$

Consequently, if $k$ is even, then $q=11$. Similarly, if $k$ is odd, then $11 \mid x$ and $p=11$. If $q=11$, we have $\left|s^{2} \mp 11^{n+1} s-11^{2 n}\right|=1$, and

$$
s=\frac{1}{2}\left( \pm 11^{n+1} \pm \sqrt{11^{2 n+2}-4\left(-11^{2 n} \mp 1\right)}\right) .
$$

This implies that there is a $\lambda \in \mathbb{Z}$ such that $\lambda^{2}=125 \times 11^{2 n} \pm 4$. However, $\lambda^{2}+4=125 \times 11^{2 n}$ is not solvable by considering it mod 11 , as then $\lambda^{2} \equiv-4$ mod 11 which contradicts that the Legendre symbol $\left(\frac{-4}{11}\right)=-1$. Moreover, $\lambda^{2}-4=125 \times 11^{2 n}$ can be shown to be non-solvable because $\lambda-2$ and $\lambda+2$ are coprime. Thus $\lambda-2 \in\left\{ \pm 1, \pm 125, \pm 11^{2 n}, \pm 125 \times 11^{2 n}\right\}$, and the only possible value for $|s|$ is 122 , which is not a prime power. An identical argument holds when $p=11$.

Now we solve the equation $x^{2}-125 y^{2}=4$. When $q$ is odd, one has that $x-2$ and $x+2$ are coprime. Following the same argument in the previous paragraph, $|s|$ cannot be a prime power. Now assume $q=2$. We divide by 4 and obtain the new Diophantine equation $x^{2}-125 \times 2^{2 n-2}=1$ or $(x-1)(x+1)=125 \times 2^{2 n-2}$, and 
$x-1 \in\left\{ \pm 2^{2 n-h-2} \times 125,2^{2 n-h-2}: h>0\right\}$. Consequently, either $x+1= \pm 2^{h}=$ $\pm 2^{2 n-h-2} \times 125+2$, or $x+1= \pm 2^{h} \times 125=2^{2 n-h-2}+2$. Therefore, $h=1$ or $2 n-h-1=1$, which is a contradiction in both cases.

Theorem 3.4. Let $E / \mathbb{Q}$ be an elliptic curve such that $E(\mathbb{Q})[5] \neq\{0\}$. Assume moreover that $N_{E}=p^{\alpha} q^{\beta}$, where $p \neq q$ are primes, and $\alpha, \beta>0$. Then $\left|\Delta_{E}\right|<N_{E}^{6}$. In particular, $E / \mathbb{Q}$ satisfies Szpiro's conjecture.

Proof. We check that $\left|\Delta_{E}\right|<N_{E}^{6}$ for the possible values of $\Delta_{E}$ given in Theorem 3.3. In fact, this is clear except when $\left|\Delta_{E}\right|=p^{5 k} q$.

In the proof of Theorem 3.3. we observe that $q=\left|p^{2 k} \mp 11 p^{k}-1\right|$. In fact, $p^{2 k} \mp 11 p^{k}-1>p^{k}$ when $p^{k}>11$. In the latter case

$$
\begin{aligned}
N_{E}^{6} & =p^{6}\left(p^{2 k} \mp 11 p^{k}-1\right)^{6}=p^{6}\left(p^{2 k} \mp 11 p^{k}-1\right)^{5}\left(p^{2 k} \mp 11 p^{k}-1\right) \\
& >p^{6+5 k}\left(p^{2 k} \mp 11 p^{k}-1\right)>p^{5 k} q=\left|\Delta_{E}\right| .
\end{aligned}
$$

We are left with treating a finite number of possible cases, namely

$$
p^{k} \in\{2,3,4,5,7,8,9,11\} .
$$

Straightforward calculations show that for all these cases if $N_{E}$ is a product of two distinct prime powers, then $\left|\Delta_{E}\right| \leq N_{E}^{6}$.

3.3. Case $n=6$. Let $P \in E(\mathbb{Q})[6]$. There exists a $\lambda \in \mathbb{Q}$ such that the following Weierstrass equation describes $E$ :

$$
y^{2}+(1-\lambda) x y-\lambda(\lambda+1) y=x^{3}-\lambda(\lambda+1) x^{2} .
$$

Assuming $\lambda=s / t, \operatorname{gcd}(s, t)=1$, we may use the transformation $x \mapsto x / t^{2}, y \mapsto$ $y / t^{3}$ to obtain the following Weierstrass equation:

$$
y^{2}+(t-s) x y-\left(t^{2} s+t s^{2}\right) y=x^{3}-\left(s t+s^{2}\right) x^{2},
$$

where the invariant of $E$ are given by

$$
\begin{aligned}
\Delta_{E} & =s^{6} t^{2}(s+t)^{3}(9 s+t), \\
c_{4} & =(3 s+t)\left(3 s^{3}+3 s^{2} t+9 s t^{2}+t^{3}\right) .
\end{aligned}
$$

Theorem 3.5. Let $E / \mathbb{Q}$ be an elliptic curve such that $E(\mathbb{Q})[6] \neq\{0\}$. Assume moreover that $N_{E}=p^{\alpha} q^{\beta}$, where $p \neq q$ are primes, and $\alpha, \beta>0$. Then $\Delta_{E}$ is given as follows:

$2 \times 7^{2},-2^{2} \times 7,2^{3} \times 7^{6}, 2^{4} \times 5,-2^{4} \times 3^{3}, 2^{6} \times 17,-2^{6} \times 7^{3}, 2^{8} \times 3^{3},-2^{8} \times 5^{2}$.

In particular, $\left|\Delta_{E}\right|<N_{E}^{6}$, and Szpiro's conjecture holds for $E$.

Proof. Let $s, t \in \mathbb{Z}, \operatorname{gcd}(s, t)=1$, be such that the following Weierstrass equation describes $E$ :

$$
y^{2}+(t-s) x y-\left(t^{2} s+t s^{2}\right) y=x^{3}-\left(s t+s^{2}\right) x^{2},
$$

where

$$
\operatorname{gcd}(s, t)=\operatorname{gcd}(s, s+t)=\operatorname{gcd}(t, s+t)=\operatorname{gcd}(s, 9 s+t)=1
$$

and

$$
\operatorname{gcd}(t, 9 s+t)|9, \operatorname{gcd}(s+t, 9 s+t)| 8 .
$$


Case i. Assume $\operatorname{gcd}(t, 9 s+t)=\operatorname{gcd}(s+t, 9 s+t)=1$. Then at least two of $|s|,|t|, \mid s+$ $t|| 9 s+t \mid,=1$. If $s=t= \pm 1$, then $s+t= \pm 2,9 s+t= \pm 10$, and $\Delta_{E}=2^{4} \times 5$. When $s= \pm 1, s+t=\mp 1$, one has $t=\mp 2,9 s+t= \pm 7$, and $\Delta_{E}=-2^{2} \times 7$. When $|s|=$ $|9 s+t|=1$, one has that $(s, t, 9 s+t, s+t) \in\{( \pm 1, \mp 8, \pm 1, \mp 7),( \pm 1, \mp 10, \mp 1, \mp 9)\}$, and the first quadruple yields $\Delta_{E}=-2^{6} \times 7^{3}$. When $t= \pm 1, s+t=\mp 1$, one has $s=\mp 2,9 s+t=\mp 17$, and $\Delta_{E}=2^{6} \times 17$. The possibilities $|t|=|9 s+t|=1$ and $|s+t|=|9 s+t|=1$ are rejected.

Case ii. Assume $\operatorname{gcd}(t, 9 s+t)=3^{k}$ and $\operatorname{gcd}(s+t, 9 s+t)=2^{l}$, where $1 \leq k \leq$ $2, \leq l \leq 3$. Then $|t|=3^{f},|s+t|=2^{g}$ and $|s|=1$. In other words, $\left|1 \pm 3^{f}\right|=2^{g}$. According to Proposition 2.1, the only integer solutions of the latter equation will yield the following set of quadruples:

$$
(s, t, s+t, 9 s+t) \in\left\{\left( \pm 1, \pm 3, \pm 2^{2}, \pm 12\right),( \pm 1, \mp 3, \mp 2, \pm 6),\left( \pm 1, \mp 3^{2}, \mp 2^{3}, 0\right)\right\} .
$$

The first quadruple gives $\Delta_{E}=2^{8} \times 3^{3}$, whereas the second gives $\Delta_{E}=-2^{4} \times 3^{3}$.

Case iii. Now assume $\operatorname{gcd}(s+t, 9 s+t)=2^{l}, 1 \leq l \leq 3$, and $\operatorname{gcd}(t, 9 s+t)=1$. The following table provides the possible values of the discriminant of $E$ :

\begin{tabular}{|c|c|c|}
\hline$|s+t|$ & $2^{f}$ & $2^{f} p^{m}$ \\
\hline $2^{g}$ & $-2^{8} \times 5^{2}, 2^{13} \times 7^{2}, 2^{15} \times 7^{6}$ & - \\
\hline $2^{g} q^{n}$ & $2^{4} \times 5$ & - \\
\hline
\end{tabular}

If $(|s+t|,|9 s+t|)=\left(2^{f}, 2^{g}\right)$, then $2^{g}=|9 s+t|=\left|8 s \pm 2^{f}\right|$. It follows that either $f=g=2$ or $\min (f, g)=3$. We obtain the following quadruples: $(s, t, s+t, 9 s+t) \in$ $\left\{\left( \pm 1, \pm 7, \pm 2^{3}, \pm 2^{4}\right),\left( \pm 1, \mp 5, \mp 2^{2}, \pm 2^{2}\right),\left( \pm 7, \pm 1, \pm 2^{3}, \pm 2^{6}\right)\right\}$. The first and third quadruples give non-minimal elliptic curves and so we have to minimize them. If $(|s+t|,|9 s+t|) \in\left\{\left(2^{f} p^{m}, 2^{g}\right),\left(2^{f}, 2^{g} q^{n}\right)\right\}$, then $|s|=|t|=1$ and the second pair gives $s+t= \pm 2,9 s+t= \pm 10$.

Case iv. Assume $\operatorname{gcd}(t, 9 s+t)=3^{l}, l \in\{1,2\}$, and $\operatorname{gcd}(s+t, 9 s+t)=1$. In fact, the only prime divisor of $t$ and $9 s+t$ is 3 , since otherwise $|s|=|s+t|=1$, where $t$ is divisible by 3 . Therefore, $(|t|,|9 s+t|)=\left(3^{f}, 3^{g}\right)$, where $\min (f, g) \in$ $\{1,2\}$, and $\left|\Delta_{E}\right|=s^{6} 3^{2 f}(s+t)^{3}\left( \pm 3^{g}\right)$. Either $|s|$ or $|s+t|$ is 1 . If $|s|=1$, then $3^{g}=|9 s+t|=\left| \pm 9+3^{f}\right|$ and there is no integer $s$ satisfying the latter equalities. If $|s+t|=1$, then $3^{g}=|9 s+t|=|9(s+t)-8 t|=\left|9 \pm 8 \times 3^{f}\right|$. The only quadruple $(s, t, s+t, 9 s+t)$ satisfying the latter equalities under the condition that $\min (f, g) \in\{1,2\}$ is $\left( \pm 10, \mp 3^{2}, \pm 1, \pm 3^{4}\right)$, but then $\Delta_{E}$ has three distinct prime divisors.

3.4. Case $n=7$. Let $P \in E(\mathbb{Q})[7]$. Then there exists a $\lambda \in \mathbb{Q}$ such that the following Weierstrass equation describes $E$ :

$$
y^{2}+(1-\lambda(\lambda-1)) x y-\lambda^{2}(\lambda-1) y=x^{3}-\lambda^{2}(\lambda-1) x^{2} .
$$

Theorem 3.6. Let $E / \mathbb{Q}$ be an elliptic curve such that $E(\mathbb{Q})[7] \neq\{0\}$. Assume moreover that $N_{E}=p^{\alpha} q^{\beta}$, where $p, q$ are distinct primes, and $\alpha, \beta>0$. Then $\Delta_{E}=-2^{7} \times 13$. In particular, $\left|\Delta_{E}\right|<N_{E}^{3}$, and Szpiro's conjecture holds for E.

Proof. Assuming $\lambda=s / t, \operatorname{gcd}(s, t)=1$, we use the transformation $x \mapsto x / t^{4}, y \mapsto$ $y / t^{6}$ to obtain the following integral Weierstrass equation describing $E$ :

$$
y^{2}+\left(t^{2}-s^{2}+s t\right) x y-s^{2}\left(s t^{3}-t^{4}\right) y=x^{3}-\left(s^{3} t-s^{2} t^{2}\right) x^{2}
$$


with invariants

$$
\begin{aligned}
\Delta_{E} & =s^{7} t^{7}(s-t)^{7}\left(s^{3}-8 s^{2} t+5 s t^{2}+t^{3}\right), \\
c_{4} & =\left(s^{2}-s t+t^{2}\right)\left(s^{6}-11 s^{5} t+30 s^{4} t^{2}-15 s^{3} t^{3}-10 s^{2} t^{4}+5 s t^{5}+t^{6}\right) .
\end{aligned}
$$

We set $k=s^{3}-8 s^{2} t+5 s t^{2}+t^{3}$. Then

$\operatorname{gcd}(s, t)=\operatorname{gcd}(s, s-t)=\operatorname{gcd}(s, k)=\operatorname{gcd}(t, s-t)=\operatorname{gcd}(t, k)=\operatorname{gcd}(s-t, k)=1$.

Since $\Delta_{E}$ has only two distinct prime divisors, then at least two of $|s|,|t|,|s-t|,|k|$ are ones. Indeed, if two of $|s|,|t|,|s-t|$ are ones, then the only corresponding discriminant is $\Delta_{E}=-2^{7} \times 13$.

If $|s|=|k|=1$, then $\left| \pm 1-8 t \pm 5 t^{2}+t^{3}\right|=1$ and either $t=0$ or $t=s= \pm 1$, which yields $s-t=0$. The same holds if $|t|=|k|=1$ or $|s-t|=|k|=1$.

3.5. Case $n=8$. Let $P \in E(\mathbb{Q})[8]$. Then there exists a $\lambda \in \mathbb{Q}$ such that $E$ is described by the following Weierstrass equation:

$$
y^{2}+\left(1-\frac{(2 \lambda-1)(\lambda-1)}{\lambda}\right) x y-(2 \lambda-1)(\lambda-1) y=x^{3}-(2 \lambda-1)(\lambda-1) x^{2} .
$$

Theorem 3.7. Let $E / \mathbb{Q}$ be an elliptic curve such that $E(\mathbb{Q})[8] \neq\{0\}$. Assume moreover that $N_{E}=p^{\alpha} q^{\beta}$, where $p \neq q$ are primes, and $\alpha, \beta>0$. Then $\Delta_{E}=$ $-2^{11} \times 3^{8}$. In particular, $\left|\Delta_{E}\right|<N_{E}^{7}$, and Szpiro's conjecture holds for $E$.

Proof. In the above Weierstrass equation we take $\lambda=s / t$, where $\operatorname{gcd}(s, t)=1$. Then we apply the transformation

$$
x \mapsto \frac{x}{s^{2} t^{2}}, y \mapsto \frac{y}{s^{3} t^{3}}
$$

to obtain the integral Weierstrass equation

$$
y^{2}-\left(t^{2}-4 s t+2 s^{2}\right) x y-t s^{3}(s-t)(2 s-t) y=x^{3}-s^{2}(s-t)(2 s-t) x^{2}
$$

where

$$
\begin{aligned}
\Delta_{E} & =s^{8} t^{2}(s-t)^{8}(2 s-t)^{4}\left(8 s^{2}-8 s t+t^{2}\right), \\
c_{4} & =16 s^{8}-64 s^{7} t+224 s^{6} t^{2}-448 s^{5} t^{3}+480 s^{4} t^{4}-288 s^{3} t^{5}+96 s^{2} t^{6}-16 s t^{7}+t^{8} .
\end{aligned}
$$

If $t$ is even, one has that $|s|,|t|,|s-t|$ and $|s-t / 2|$ are pairwise coprime, so at least two of these are ones. If $|s|=|s-t|=1$, then $s= \pm 1, t= \pm 2$ and $2 s-t=0$. If $|s|=|s-t / 2|=1$, then $s= \pm 1, t= \pm 4, s-t=\mp 3,8 s^{2}-8 s t+t^{2}=-8$ and $\Delta_{E}=-2^{11} \times 3^{8}$. If $s-t= \pm 1, s-t / 2=\mp 1$, then $s=\mp 3, t=\mp 4,2 s-t=$ $\mp 2,8 s^{2}-8 s t+t^{2}=-8$ and $\Delta_{E}=-2^{11} \times 3^{8}$.

If $t$ is odd, then at least two of $|s|,|t|,|s-t|$ and $|2 s-t|$ are ones. The following table contains $\left|s t(s-t)(2 s-t)\left(8 s^{2}-8 s t+t^{2}\right)\right|$

\begin{tabular}{|c|c|c|c|}
\hline & $|s|=1$ & $|t|=1$ & $|2 s-t|=1, t$ odd \\
\hline$|s-t|=1$ & $t$ even & $2 \times 3 \times 17$ & $2 \times 3 \times 7$ \\
\cline { 1 - 3 }$|2 s-t|=1, t$ odd & $2 \times 3 \times 7$ & 0 & \multicolumn{1}{|c}{} \\
\cline { 1 - 3 }$|t|=1$ & $2 \times 3 \times 17$ & \multicolumn{2}{|c}{} \\
\cline { 1 - 2 } & &
\end{tabular}

Therefore, the only elliptic curve with an 8-torsion point and whose conductor is a product of two distinct prime powers is the one with $\Delta_{E}=-2^{11} \times 3^{8}$. We observe that its invariant $c_{4}=-2^{4} \times 47$, so it has additive reduction over $\mathbb{F}_{2}$ and $N_{E}=2^{2} \times 3$. 
3.6. Case $n=9$. Let $P \in E(\mathbb{Q})[9]$. There exists a $\lambda \in \mathbb{Q}$ such that $E$ is described by the following Weierstrass equation:

$y^{2}+\left(1-\lambda^{2}(\lambda-1)\right) x y-\lambda^{2}(\lambda-1)\left(\lambda^{2}-\lambda+1\right) y=x^{3}-\lambda^{2}(\lambda-1)\left(\lambda^{2}-\lambda+1\right) x^{2}$.

Theorem 3.8. Let $E / \mathbb{Q}$ be an elliptic curve such that $E(\mathbb{Q})[9] \neq\{0\}$. Assume moreover that $N_{E}=p^{\alpha} q^{\beta}$, where $p \neq q$ are primes, and $\alpha, \beta>0$. Then $\Delta_{E}=$ $-2^{9} \times 3^{5}$. In particular, $\left|\Delta_{E}\right|<N_{E}^{5}$, and Szpiro's conjecture holds for $E$.

Proof. In the above Weierstrass equation we take $\lambda=s / t$, where $\operatorname{gcd}(s, t)=1$. Then we apply the transformation

$$
x \mapsto \frac{x}{t^{6}}, y \mapsto \frac{y}{t^{9}}
$$

to obtain the following integral Weierstrass equation describing $E$ :

$y^{2}+\left(t^{3}-s^{2}(s-t)\right) x y-t^{4} s^{2}(s-t)\left(s^{2}-s t+t^{2}\right) y=x^{3}-t s^{2}(s-t)\left(s^{2}-s t+t^{2}\right) x^{2}$,

where

$$
\begin{aligned}
\Delta_{E}= & s^{9} t^{9}(s-t)^{9}\left(s^{2}-s t+t^{2}\right)^{3}\left(s^{3}-6 s^{2} t+3 s t^{2}+t^{3}\right), \\
c_{4}= & \left(s^{3}-3 s^{2} t+t^{3}\right)\left(s^{9}-9 s^{8} t+27 s^{7} t^{2}-48 s^{6} t^{3}+54 s^{5} t^{4}\right. \\
& \left.\quad-45 s^{4} t^{5}+27 s^{3} t^{6}-9 s^{2} t^{7}+t^{9}\right) ;
\end{aligned}
$$

since $s^{2}-s t+t^{2}=(s-t)^{2}+s t$, the first four factors $s, t, s-t, s^{2}-s t+t^{2}$ of $\Delta_{E}$ are pairwise coprime. Therefore, at least two of the absolute values of these factors are ones. If $s= \pm 1, t=\mp 1$, then $s-t= \pm 2, s^{2}-s t+t^{2}=3, s^{3}-6 s^{2} t+3 s t^{2}+t^{3}= \pm 9$, $\Delta_{E}=-2^{9} \times 3^{5}$. If $|s|=|s-t|=1$, then $s= \pm 1, t= \pm 2, s-t=\mp 1, s^{2}-s t+t^{2}=3$, $s^{3}-6 s^{2} t+3 s t^{2}+t^{3}= \pm 9, \Delta_{E}=-2^{9} \times 3^{5}$. If $s= \pm 1,-1=s^{2}-s t+t^{2}=$ $1 \mp t+t^{2}$, then there is no integer $t$ satisfying the latter equalities. If $s= \pm 1$, $\left|s^{3}-6 s^{2} t+3 s t^{2}+t^{3}\right|=1$, then $t= \pm 1$. We will have the same results if we replace $|s|=1$ by $|t|=1$. If $|s-t|=1$ and $\left|s^{2}-s t+t^{2}\right|=\left|(s-t)^{2}+s t\right|=|1+s t|=1$, then $s t=-2$, a contradiction.

The only elliptic curve with a 9-torsion point and whose conductor is a product of two distinct prime powers is the one with $\Delta_{E}=-2^{9} \times 3^{5}$. We observe that $\operatorname{ord}_{3}\left(c_{4}\right)>0$, so it has additive reduction over $\mathbb{F}_{3}$ and $N_{E}=2 \times 3^{2}$.

3.7. Case $n=10$. Let $P \in E(\mathbb{Q})[10]$. There exists a $\lambda \in \mathbb{Q}$ such that the following Weierstrass equation describes $E$ :

$y^{2}+\left(1+\frac{\lambda(\lambda-1)(2 \lambda-1)}{\left(\lambda^{2}-3 \lambda+1\right)}\right) x y-\frac{\lambda^{3}(\lambda-1)(2 \lambda-1)}{\left(\lambda^{2}-3 \lambda+1\right)^{2}} y=x^{3}-\frac{\lambda^{3}(\lambda-1)(2 \lambda-1)}{\left(\lambda^{2}-3 \lambda+1\right)^{2}} x^{2}$.

Theorem 3.9. There exists no elliptic curve $E / \mathbb{Q}$ with $E(\mathbb{Q})[10] \neq\{0\}$ and $N_{E}=$ $p^{\alpha} q^{\beta}$, where $p \neq q$ are primes, and $\alpha, \beta>0$.

Proof. In the above Weierstrass equation we take $\lambda=s / t$, where $\operatorname{gcd}(s, t)=1$. Then we apply the transformation

$$
x \mapsto \frac{x}{t^{2}\left(s^{2}-3 s t+t^{2}\right)^{2}}, y \mapsto \frac{y}{t^{3}\left(s^{2}-3 s t+t^{2}\right)^{3}}
$$

to obtain the integral Weierstrass equation

$$
\begin{array}{r}
y^{2}+\left[t\left(s^{2}-3 s t+t^{2}\right)+s(s-t)(2 s-t)\right] x y-t^{2} s^{3}(s-t)(2 s-t)\left(s^{2}-3 s t+t^{2}\right) y \\
=x^{3}-t s^{3}(s-t)(2 s-t) x^{2}
\end{array}
$$


where

$$
\Delta_{E}=s^{10} t^{5}(s-t)^{10}(2 s-t)^{5}\left(4 s^{2}-2 s t-t^{2}\right)\left(s^{2}-3 s t+t^{2}\right)^{2} .
$$

The factors $s, t, s-t, s^{2}-3 s t+t^{2}$ are pairwise coprime. Therefore, at least two of $|s|,|t|,|s-t|,\left|s^{2}-3 s t+t^{2}\right|$ are ones. The following table contains the product $\left|s t(s-t)(2 s-t)\left(4 s^{2}-2 s t-t^{2}\right)\left(s^{2}-3 s t+t^{2}\right)\right|$ when two of $|s|,|t|,|s-t|,\left|s^{2}-3 s t+t^{2}\right|$ are ones:

\begin{tabular}{|c|c|c|c|c|c|}
\hline & $|s|=1$ & $|t|=1$ & $|s-t|=1$ & $|2 s-t|=1$ & $\left|4 s^{2}-2 s t-t^{2}\right|=1$ \\
\hline$\left|s^{2}-3 s t+t^{2}\right|=1$ & 66 & 870,66 & 0,66 & $870,66,0$ & 0 \\
\hline$\left|4 s^{2}-2 s t-t^{2}\right|=1$ & 60 & 0 & 60 & 0 & \\
\hline$|2 s-t|=1$ & 66 & 0 & 30 & & \\
\hline$|s-t|=1$ & 0 & 66 & & & \\
\hline$|t|=1$ & 30 & & & & \\
\hline
\end{tabular}

It is clear that $E$ cannot have a discriminant with only two distinct prime divisors.

3.8. Case $n=12$. Let $P \in E(\mathbb{Q})[12]$. There exists a $\lambda \in \mathbb{Q}$ such that the following Weierstrass equation describes $E$ :

$$
\begin{aligned}
y^{2} & +\left(1+\frac{\lambda(2 \lambda-1)\left(3 \lambda^{2}-3 \lambda+1\right)}{(\lambda-1)^{3}}\right) x y-\frac{\lambda(2 \lambda-1)\left(3 \lambda^{2}-3 \lambda+1\right)\left(2 \lambda^{2}-2 \lambda+1\right)}{(\lambda-1)^{4}} y \\
& =x^{3}-\frac{\lambda(2 \lambda-1)\left(3 \lambda^{2}-3 \lambda+1\right)\left(2 \lambda^{2}-2 \lambda+1\right)}{(\lambda-1)^{4}} x^{2} .
\end{aligned}
$$

Theorem 3.10. There exists no elliptic curve $E / \mathbb{Q}$ with $E(\mathbb{Q})[12] \neq\{0\}$ and $N_{E}=p^{\alpha} q^{\beta}$, where $p \neq q$ are primes, and $\alpha, \beta>0$.

Proof. In the above Weierstrass equation we take $\lambda=s / t$, where $\operatorname{gcd}(s, t)=1$. Then we apply the transformation

$$
x \mapsto \frac{x}{t^{2}(s-t)^{6}}, y \mapsto \frac{y}{t^{3}(s-t)^{9}}
$$

to obtain the integral Weierstrass equation

$$
\begin{aligned}
y^{2}+ & {\left[t(s-t)^{3}+s(2 s-t)\left(3 s^{2}-3 s t+t^{2}\right)\right] x y } \\
& -t s(s-t)^{5}(2 s-t)\left(3 s^{2}-3 s t+t^{2}\right)\left(2 s^{2}-2 s t+t^{2}\right) y \\
& =x^{3}-s(s-t)^{2}(2 s-t)\left(3 s^{2}-3 s t+t^{2}\right)\left(2 s^{2}-2 s t+t^{2}\right) x^{2},
\end{aligned}
$$

where

$$
\Delta_{E}=s^{12} t^{2}(s-t)^{12}(2 s-t)^{6}\left(3 s^{2}-3 s t+t^{2}\right)^{4}\left(2 s^{2}-2 s t+t^{2}\right)^{3}\left(6 s^{2}-6 s t+t^{2}\right)
$$

If $t$ is even, then two of $|s|,|t|,|s-t|$ and $|s-t / 2|$ are ones. If $t$ is odd, then two of $|s|,|t|,|s-t|$ and $|2 s-t|$ are ones. In both cases, one finds that the product corresponding to $\Delta_{E}$ is either 0 or has more than two prime divisors.

\section{ACKNOWLEDGEMENTS}

The author would like to thank Michael Bennett for spotting out a mistake in the proof of Theorem 3.3. He would also like to thank Andrzej Dabrowski for sharing some of the results of his current work on elliptic curves with small conductor. 


\section{REFERENCES}

[1] Yu. Bilu, G. Hanrot, and P. M. Voutier, Existence of primitive divisors of Lucas and Lehmer numbers, J. Reine Angew. Math. 539 (2001), 75-122, DOI 10.1515/crll.2001.080. With an appendix by M. Mignotte. MR.1863855(2002j:11027)

[2] Yann Bugeaud, Maurice Mignotte, and Yves Roy, On the Diophantine equation $\left(x^{n}-\right.$ 1) $/(x-1)=y^{q}$, Pacific J. Math. 193 (2000), no. 2, 257-268, DOI 10.2140/pjm.2000.193.257. MR:1755817 (2001f:11049)

[3] Zhenfu Cao, Chuan I. Chu, and Wai Chee Shiu, The exponential Diophantine equation $A X^{2}+B Y^{2}=\lambda k^{Z}$ and its applications, Taiwanese J. Math. 12 (2008), no. 5, 1015-1034. MR2431876(2009d:11058)

[4] Henri Cohen. Number theory, Volume I: Tools and Diophantine equations. Graduate Texts in Mathematics 239. Springer-Verlag, 2007. MR2312337

[5] J. Gebel, A. Pethö, and H. G. Zimmer, On Mordell's equation, Compositio Math. 110 (1998), no. 3, 335-367, DOI 10.1023/A:1000281602647. MR1602064 (98m:11049)

[6] Toshihiro Hadano, Remarks on the conductor of an elliptic curve, Proc. Japan Acad. 48 (1972), 166-167. MR0311668 (47 \#230)

[7] Toshihiro Hadano, On the conductor of an elliptic curve with a rational point of order 2, Nagoya Math. J. 53 (1974), 199-210. MR0354673 (50 \#7151)

[8] D. Lorenzini, Torsion and Tamagawa numbers, Ann. Inst. Fourier 61 (2011), no. 5, 19952037. MR2961846

[9] A. P. Ogg, Abelian curves of 2-power conductor, Proc. Cambridge Philos. Soc. 62 (1966), 143-148. MR0201436 (34 \#1320)

[10] A. P. Ogg, Abelian curves of small conductor, J. Reine Angew. Math. 226 (1967), 204-215. MR0210706 (35 \#1592)

[11] Alice Silverberg, Open questions in arithmetic algebraic geometry, Arithmetic algebraic geometry (Park City, UT, 1999), IAS/Park City Math. Ser., vol. 9, Amer. Math. Soc., Providence, RI, 2001, pp. 83-142. MR1860041 (2002g:11073)

[12] Joseph H. Silverman, The arithmetic of elliptic curves, Graduate Texts in Mathematics, vol. 106, Springer-Verlag, New York, 1986. MR817210 (87g:11070)

[13] Joseph H. Silverman, Advanced topics in the arithmetic of elliptic curves, Graduate Texts in Mathematics, vol. 151, Springer-Verlag, New York, 1994. MR.1312368 (96b:11074)

Department of Mathematics and Actuarial Science, American University in Cairo, New Cairo, Egypt 11835

E-mail address: mmsadek@aucegypt.edu 